\title{
Le bousillage. Détournements et appropriations des techniques et des outils de production chez les ouvriers verriers à la fin du XIX siècle
}

Whimsies. Factory homing and the appropriation of techniques and production tools among glass workers in the late $19^{\text {th }}$ century.

Geschundenes Glas. Unterschlagung und Aneignung von Produktionstechniken und Werkzeugen bei den Glasmacherarbeitern am Ende des 19. Jahrhunderts

\section{Stéphane Palaude}

\section{CpenEdition}

Journals

Édition électronique

URL : http://journals.openedition.org/rh19/4342

DOI : $10.4000 /$ rh 19.4342

ISSN : $1777-5329$

Éditeur

La Société de 1848

Édition imprimée

Date de publication : 31 décembre 2012

Pagination : 111-126

ISSN : 1265-1354

Référence électronique

Stéphane Palaude, «Le bousillage. Détournements et appropriations des techniques et des outils de production chez les ouvriers verriers à la fin du XIXe siècle », Revue d'histoire du XIXe siècle [En ligne], 45 | 2012, mis en ligne le 31 décembre 2015, consulté le 19 avril 2019. URL : http:// journals.openedition.org/rh19/4342 ; DOI : 10.4000/rh19.4342 


\section{STÉPHANE PALAUDE}

\section{Le bousillage. Détournements et appropriations des techniques et des outils de production chez les ouvriers verriers à la fin du XIXe siècle}

Dans bien des industries, «faire de la perruque» est une activité répandue en France aux XIX ${ }^{\mathrm{e}}$ et $\mathrm{XX}^{\mathrm{e}}$ siècles. Il existe pour les ouvriers une tolérance - non reconnue officiellement par les chefs d'entreprise - à détourner une infime partie des matériaux de l'atelier et de les façonner avec le matériel du lieu de travail selon les occasions. Si la perruque de métal est connue, celle de verre l'est beaucoup moins ${ }^{1}$. Elle prend en cette circonstance la dénomination de bousillage avec cette différence fondamentale qu'il s'agit bien de verre, mais travaillé sur le temps libre du verrier. Sa concrétisation au travers de nombreux bousillages - le terme désigne à la fois l'objet et l'action - commence à fleurir dans les logements des ouvriers verriers à partir du dernier tiers du XIX ${ }^{e}$ siècle. Et que ce soit en gobeleterie (service de table), bouteillerie, flaconnage voire verre à vitres, le verrier fait montre d'une grande imagination, tant que la pièce fabriquée differe de la production habituelle de l'établissement dans lequel il est employé. Et si l'objet en verre est "détourné», il n'en garde pas moins une fonction. Or, aujourd'hui, seuls quelques amateurs éclairés comprennent ce que bousillage signifie. Pour la majorité de nos contemporains, à tort, cela rime davantage avec destruction plutôt qu'avec création. Des traces de ces pratiques subsistent : quantité de pièces de bousillage constituent des collections à part entière au musée du verre de Charleroi (Belgique) ou au musée-atelier départemental du verre à Sars-Poteries (Nord, France).

\footnotetext{
1. Sur la perruque de métal, voir notamment : Robert Kosmann, "Perruque et bricolage ouvrier», in Françoise Odin et Christian Thuderoz, Des mondes bricolés? Arts et sciences à l'épreuve de la notion de bricolage, Lausanne, Presses polytechniques et universitaires romandes, Collection «METIS LyonTech", 2010, p. 159-174 et Étienne de Banville, L'usine en douce, Le travail en "perruque», Paris, L'Harmattan, Mémoires de travail, 2001.
} 


\section{DES PRATIQUES MÉCONNUES}

Présenter le bousillage, c'est en premier lieu tenter de remédier à ce manque d'intérêt, en France, pour cette pratique verrière de la part de l'historien, à l'inverse de l'ethnologue et du sociologue.

D'une manière générale, si l'art du verre et son industrie commencent à être relativement bien traités d'un point de vue historique, ce n'est pas le cas de l'homme du verre et de son bousillage. Exception faite du présent auteur pour les verriers d'Avesnois-Thiérache (Nord-Aisne), que ce soit Yves Tyl pour ceux d'Alsace, Yves Lequin pour ceux du Lyonnais, Joan Wallach Scott pour ceux de Carmaux (Tarn), Jean Merley pour ceux de Veauche (Loire), Jean-Pierre Daviet à propos des maitres de verreries du Nord, ou encore indirectement Caroline Moriceau au travers des douleurs de l'industrie, aucun ne traite de cette pratique ${ }^{2}$. Ceux-ci ne pouvaient trouver secours dans les ouvrages antérieurs puisque, parmi les plus réputés, celui de Georges Bontemps demeure muet à ce sujet ${ }^{3}$. Il en va également ainsi pour les sources archivistiques. Seuls d'infimes détails éparpillés mentionnent le bousillage, et encore cette dénomination n'est-elle pas directement employée; ce qui n’a donc pu susciter l'intérêt du chercheur.

Une première approche apparait toutefois en 1992 sous la direction de Denis Woronoff dans Le souffle et la marque, circulation des savoirs et formation des cultures verrières ${ }^{4}$. Il aurait été intéressant de poursuivre en ce sens et d'approfondir, par exemple, le remarquable travail d'Elisabeth Cibot-Genin dans lequel le bousillage se devine par le biais de la «création et de l'innovation verrière chez Baccarat et Daum entre 1890 et $1990 »^{5}$. Les notions de création et d'innovation personnelles demeurent assez peu identifiées dans le monde verrier de la fin du XIX ${ }^{\mathrm{e}}$ siècle. Ainsi, en 1883, Georges Duponchel, maître de la verrerie d'en-bas de Sars-Poteries militant pour la création

2. Stéphane Palaude, Verreries noires d'Avesnois-Thiérache, $X I X^{e}-X X^{e}$ siècles, Thèse de doctorat en histoire sous la direction de Jean-François Eck, Université de Lille III, 2009; Yves Tyl, Le travail des enfants au $X I X^{e}$ siècle, Une région : l'Alsace, Un métier : la verrerie, thèse d'histoire sous la direction de Jacques Perot, Université de Paris VII, 1987; Yves Lequin, Les Ouvriers de la région lyonnaise (1848-1914), Les intérêts de classe et la République, Lyon, Presses universitaires de Lyon, 1977; Joan Wallach Scott, Les verriers de Carmaux. Histoire de la naissance d'un syndicalisme, Paris, Flammarion, 1982; Jean Merley, La verrerie $B S N$ de Veauche, histoire d'une entreprise forézienne, Le Puy-en-Velay, Université de Saint-Étienne, Centre interdisciplinaire d'études et de recherches sur les structures régionales, 1983; Jean-Pierre Daviet, "De la première à la seconde industrialisation : les maîtres de verreries du département du Nord au XIX ${ }^{\mathrm{e}}$ siècle", Revue $d u$ Nord, tome 67, n² 265, avril-juin 1985, p. 447-483; Caroline Moriceau, Les douleurs de l'industrie, L'hygiénisme industriel en France, 1860-1914, Paris, Éditions de l'EHESS, 2009. 3. Georges Bontemps, Guide du verrier, Paris, Librairie du dictionnaire des arts et manufactures, 1868. 4. Denis Woronoff [dir.], Le souffle et la marque, Circulation des savoirs et formation des cultures verrières, étude conduite par la Mission du patrimoine ethnologique et de la sous-direction de l'Inventaire général, de la documentation et de la protection du patrimoine, Direction du Patrimoine, Laboratoire de recherche sur le patrimoine français, unité mixte 22/CNRS, 1992.

5. Elisabeth Cibot-Genin, "Création et innovation chez Baccarat et Daum entre 1890 et 1990 ", in Le souffle et la marque..., op. cit., p. 164-276. Ce travail avait été précédé d'un mémoire que nous n'avons pu consulter : Elisabeth Cibot-Genin, Création artistique et innovation dans l'industrie verrière en France, 1880-1989, DEA d'histoire des techniques sous la direction de Denis Woronoff, Paris, EHESS, 1989. 
d'un cours de dessin destiné au personnel verrier afin qu'il puisse à son tour "innover un modèle nouveau ayant un cachet artistique», estime que l'ouvrier ne fait que reproduire les formes qu'il a apprises à modeler au cours de son apprentissage ou que son chef d'atelier lui donne par la suite à exécuter. Il reconnaît que "quelques-uns néanmoins ont un goût inné et, par tâtonnements, arrivent à de jolis résultats ${ }^{6}$. D'évidence, quelques verriers sarséens s'adonnent donc au bousillage; première confirmation française d'une existence reconnue, mais dépourvue de dénomination.

Le terme de bousillage en lui-même n'apparaît qu'à l'extrême fin du $\mathrm{XIX}^{\mathrm{e}}$ siècle et ne passe à la postérité que par la création du musée du verre de Sars-Poteries en 1967, musée dont les collections sont alors entièrement consacrées à ce "détournement". À partir de là, nombre d'érudits locaux à travers la France mettront à l'honneur cette pratique désormais reconnue, mais insuffisamment prise en compte par les historiens, sans doute parce qu'il s'agit avant tout d'une mémoire orale et de gestes.

Il faut dire que même à l'époque, peu de témoins ont vu des verriers bousiller. Leur attention est avant tout captée par la voltige du verre chaud. Bien des observateurs - et jusqu'à nous - ont d'ailleurs totalement laissé de côté l'homme, ne se focalisant que sur le travail du verre, quand ils en comprenaient toutes les étapes de fabrication. En 2010, Robert Kosmann intègre le bousillage dans sa présentation sur la perruque et le bricolage ouvrier en synthétisant des travaux précurseurs ${ }^{7}$. Car ce sont à l'ethnologue Noëlle Gérôme puis au sociologue Étienne de Banville qu'il convient de rendre l'antériorité d'une réelle recherche sur la question. La première, avec Agnès Guiche, avait étudié le monde des verriers de verre à vitres de Saint-Just-sur-Loire (Loire) au début des années 1980. Elle use d'ailleurs en 1987 du terme de «bousillé» qu'elle assimile à "bricole» ou "perruque ${ }^{8}$. Reprise dans l'excellent ouvrage d'Étienne de Banville, L'usine en douce (2001), l'étude du bousillage verrier adjectif redondant puisque le bousillage ne peut être que verrier - se trouve amplifiée, quoique partant sur une base tronquée. Selon ce dernier, perruque désigne « un travail, un objet pour soi, réalisé durant le temps de travail, avec les matériaux et le matériel de l'entreprise », parlant même plus loin d'activité semi-clandestine ${ }^{9}$. Or, c'est méconnaitre le processus de fabrication du verre.

6. «Déposition de M. Duponchel», séance du 5 juin 1883, in ministère de l'Instruction publique et des beaux-arts, Commission d'enquête sur la situation des ouvriers et des industries d'art, Paris, A. Quantin, 1884, p. 472 .

7. Robert Kosmann, «Perruque et bricolage ouvrier»... art. cit.

8. Agnès Guiche, La danse du verre, Les représentations d'une identité au travail, rapport pour la Mission du patrimoine ethnologique du ministère de la culture sous la direction de Noëlle Gérôme, juin 1985. Noëlle Gérôme et Agnès Guiche, La danse du verre. Étude avec les verriers de Saint-Just. Perception d'une identité, . Sauvegarde d'une profession, rapport de fin de contrat à la Mission du patrimoine du ministère de la culture, Fédération nationale Travail et Culture, décembre 1985. Noëlle Gérôme, «Les verriers de Saint-Just-sur-Loire. La demande d'une histoire ouvrière", Le monde alpin et rhodanien, nº 3-4, 1987, p. 213.

9. Étienne de Banville, L’usine en douce... op. cit., p. 5 et 49. 
S'il s'agit effectivement d'un objet confectionné avec les matériaux et le matériel du lieu de travail, dans le cas d'un mécanisme automatique de production, il n'y a pas de pause de fabrication : la machine fonctionnant en continu, à l'instar de celle des verriers interrogés par Étienne de Banville, le bousillage, du moins ce qu'il en est advenu par la force de la mécanisation, s'effectue effectivement sur le temps de fonctionnement de l'unité de production, donc de travail. Mais auparavant, c'était l'homme qui manipulait le verre en fusion. Le bousillage se façonnait, d'une part, hors du temps de travail, soit pendant la pause, soit en fin de journée, d'autre part, à la vue de la hiérarchie puisque tout objet en verre doit être recuit, c'est-à-dire refroidi lentement dans une chaleur dégressive et contrôlée, sinon il risque de se briser sous l'action des tensions internes. Lorsque le bousillage atteignait le terme de sa recuisson, il passait obligatoirement entre les mains du releveur d'arche de recuit et était donc à la portée de connaissance des chefs. Il ne pouvait donc être question de fabrication «en douce». Cela est encore vrai aujourd'hui, le bousillage auprès d'une machine automatique nécessitant l'accord plus ou moins tacite de la hiérarchie puisqu'il ne passe pas inaperçu au recuit. Ainsi, inutile de développer des logiques de contrôle comme dans l'industrie textile, le mode opératoire de mise en forme du verre se prêtant à une surveillance facile.

Les sources des pratiques de bousillage sont essentiellement orales. De ce fait, davantage que les historiens, ce sont les ethnologues puis les sociologues qui s'y sont intéressés, quoique leur approche pêche par un manque de connaissance du processus de fabrication. Le terme qui désigne la pratique prête lui-même à confusion.

\section{UNE DÉSIGNATION PARADOXALE}

La langue peut expliquer le manque d'intérêt des chercheurs pour le bousillage. Le terme en lui-même est passé dans les usages de la langue française en un sens contraire, synonyme de destruction plutôt que de création.

Pourquoi "bousiller »? Ce verbe se rencontre dès le XVI siècle en maçonnerie. En 1554, à la lecture des comptes de Diane de Poitiers, nous comprenons que bousiller signifie "construire en torchis» ${ }^{10}$. En 1690, outre ce sens premier communément admis, Antoine Furetière en ajoute un second par extension : il se «dit proverbialement \& par mépris des logis bâtis de mauvais matériaux, \& de plusieurs manufactures $\&$ besognes malfaites, qu'elles ne sont que bousillées ${ }^{11}$. Sur cette lancée, à la veille de Révolution,

10. Comptes de Diane de Poitiers, 181, Delb. in QUEM.; http//www.cnrtl.fr/definition/bousiller au 13 février 2012.

11. Basnage de Bauval, Dictionnaire universel [...] par feu Antoine Furetière, 2e édition revue, corrigée et augmentée, La Haye/Rotterdam, Arnoud et Reinier Leers, 1701, tome 2, non paginé. 
l'encyclopédie Panckoucke présente le bousilleur comme «celui qui travaille en bousillage [et/ou] encore un mauvais ouvrier qui [f]ait mal son métier, $\&$ qui travaille avec peu d'adresse et de propreté» ${ }^{12}$. Cet définition péjorative s'amplifie au XIX' siècle.

En 1828, bousillage «se dit figurément des ouvrages des artisans faits sans soin, à la hâte ou sans intelligence». En 1836, bousiller, c'est «travailler sans goût, gâter sa besogne». En 1869, le bousillage correspond à «tout ouvrage mal fait ou qui doit durer peu ". Au début des années 1880, bousiller, c'est "travailler grossièrement». En 1894, l'auteur du Dictionnaire d'argot finde-siècle va jusqu'à en faire le synonyme de "saboter ${ }^{13}$. L'assimilation de bousillage à un mauvais travail trouve un développement chez Paul LeroyBeaulieu en 1914 : "par l'appât d'une rémunération proportionnelle à la production, [le travail aux pièces] risque de rendre l'ouvrier moins soigneux, de provoquer chez lui, sinon des malfaçons, du moins un défaut de complet achèvement et de fini, ce que les Anglais appellent scamping, le bousillage $»^{14}$. Mais pour le monde de la production verrière, la remarque n'est pas valable : un manque de fini sur le verre se voit au relevage d'arche de recuit et la pièce est immédiatement rebutée ou déclassée. En parallèle, trente ans plus tôt, bousiller avait pris un autre sens.

Le Dictionnaire des synonymes de la langue française publié en 1884 propose un article intitulé "carnage, boucherie, massacre, tuerie», terminé par cette assertion : «massacre emporte une idée de confusion, de mélange, de désordre. On appelle massacre un ouvrier qui bousille, qui gâte la matière, faute de savoir lui donner une forme convenable $»^{15}$. Serait-ce de là que naît cette usage ultérieur, pour bousiller, de tuer, détruire - interprétation si chère aux Poilus de la Grande guerre ${ }^{16}$ ? Tout naturellement, nos contemporains de l'aube du XXI e siècle assimilent bousillage à destruction. Au point que fin 2010, au musée du verre de Charleroi, Rina Margos, coordinatrice de l'exposition cédant à la pression de son administration, substituera à Bousillage le titre Les verriers de l'ombre ou le Souffle populaire'17. Or, le bousillagedestruction ne correspond en rien à la réalité du bousillage-création que représente la lampe du mariage Imbert-Deharveng de Sars-Poteries réalisée

12. Encyclopédie méthodique. Commerce, Paris, Panckoucke, 1783-1784, tome 1, p. 298.

13. J.-Ch. Laveaux, Nouveau dictionnaire de la langue française, seconde édition, Paris, Deterville, 1828, p. 253. Babault, Dictionnaire français et géographique..., $A$-C, Paris, chez l'auteur, 1836, tome 1, p. 362; Maurice Lachâtre, Nouveau dictionnaire universel, Paris, F. Cantel, 1869, tome 1, p. 641; Alphonse Ardoin, Le Dictionnaire des écoles, Paris, Grandremy et Hénon, 1880-1881, p. 139; Charles Virmaître, Dictionnaire d'argot fin-de-siècle, Paris, A. Charles, 1894, p. 46.

14. Paul Leroy-Beaulieu, Traité théorique et pratique d'économie politique, Paris, Félix Alcan, 1914, tome 2, p. 265.

15. B. Lafaye, Dictionnaire des synonymes de la langue française, Paris, Hachette, 1884, p. 423.

16. A. Dauzat, L'argot de la Guerre, d'après une enquête auprès des officiers et soldats, Paris, Armand Colin, 2007 [1918], p. 221-222.

17. Rina Margos et Virginia Colom Izquierdo [dir.], Les verriers de l'ombre ou le Souffle populaire, catalogue de l'exposition, Musée du verre, Bois du Cazier, Marcinelle, Charleroi, 11 septembre - 7 novembre 2010, Couillet, Antoine Tanzilli, 2010. 


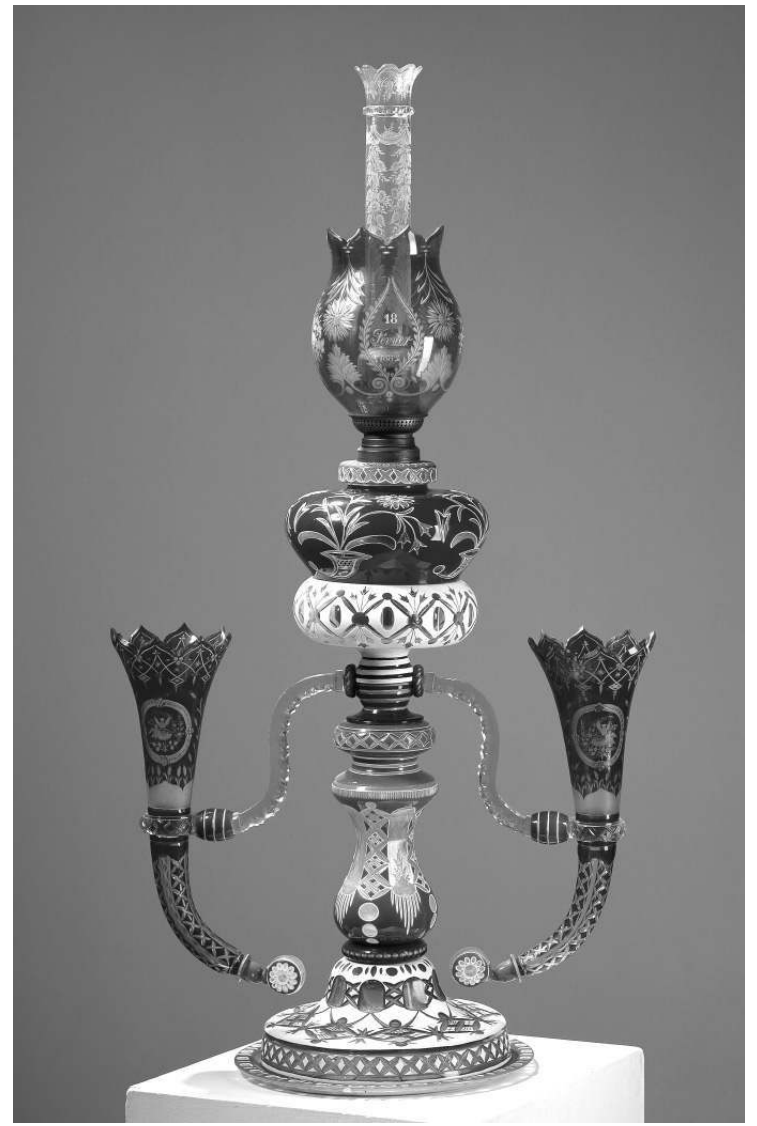

Fig. 1 : Lampe à pétrole de prestige bousillée offerte par les ouvriers verriers de la verrerie d'en-haut de Sars-Poteries à l'occasion du mariage de leur maître de verreries, HenriLéon Imbert, avec $\mathrm{M}^{\text {lle }}$ Marie Deharveng le 18 février 1882; Florent Vinck père \& fils (soufflage) Joseph Poels (gravure et taille).

Collection du Musée-atelier départemental du verre à SarsPoteries - Conseil Général du Nord, inv. ${ }^{\circ}$ 967.4.13.

Cliché () Paul Louis.

en 1882 (fig. 1). Loin des considérations du compagnonnage, lequel n'existe pas en verrerie, il est ici possible de parler de chef-d'œuvre exceptionnel de par sa qualité d'exécution, les souffleurs ayant joué de la superposition des couches de verre dans une variété de combinaisons que le tailleur n'a pas manqué de faire ensuite ressortir, et le graveur ayant ensuite travaillé certaines parties.

Du torchis au verre, le pas est grand, plus encore celui qui va de la destruction à la création. Le bousillage exécuté pour le couple Imbert-Deharveng ne doit cependant pas cacher tous ceux façonnés pour un usage plus personnel par des générations «d'ouvriers en verre».

\section{UN DÉTOURNEMENT «SUR PLACE »}

Le bousillage fleurit à partir du dernier tiers du XIX siècle dans les verreries. Est-ce véritablement un détournement? Où se pratique-t-il? Selon Noëlle Gérôme, pour qu'il y ait détournement, cela nécessite la non-maitrise 
de la procédure de travail. Il faut une norme à transgresser ${ }^{18}$. Ce qui est ici le cas. Et celle-ci d'ajouter que, d'une manière générale, le patronat tolère cette pratique pour entretenir la compétence et la virtuosité de ses ouvriers. Pouvons-nous compléter cette vision par la transmission du savoir-faire? En effet, dans le dernier tiers du XIX ${ }^{\mathrm{e}}$ siècle, l'apprentissage en verrerie n'est le fruit que de la répétition. Comment? Même s'il est impossible de définir une équipe modèle, la "place» - à la fois équipe et lieu d'exercice du travail - se compose de trois à cinq personnes en flaconnage comme en bouteillerie, de trois à six en verrerie de verre à vitres et, en gobeleterie, de trois personnes pour le formage à la presse et de quatre à huit pour le soufflage à la bouche. L'attribution d'une tâche précise et répétitive à chacun optimise la productivité de l'équipe. Pour acquérir le savoir-faire, point d'école mais un apprentissage qui s'effectue "sur le tas" et nécessite sept à dix ans de tâtonnements dans la manipulation d'un matériau incandescent, manipulation de l'instant qu'il est impossible de reporter, sous peine de tout recommencer. Si le mot tâtonnement paraît fort, il traduit la réalité. C'est en observant que le futur verrier apprend; la "place» étant payée au nombre de pièces, son chef ne peut suspendre le travail pour former un apprenti.

Pour la pratique, il est d'usage de faire permuter chaque membre de l'équipe en toute fin de journée ${ }^{19}$. Le porteur à l'arche de recuit devient batteur de cannes, le batteur, cueilleur, etc. Justement, chez les verriers anglosaxons, le bousillage est dénommé after hour glass, verre après l'heure de travail, ou encore end of day glass, verre de fin de journée de travail. Car l'autre solution de l'apprentissage, c'est le bousillage, lequel, selon sa propre définition, correspond à l'action et à l'objet en verre façonné, d'une part, hors du temps réglementaire de travail et, d'autre part, selon des modèles qui ne peuvent entrer en compétition avec la production de l'établissement dans lequel le verrier est employé. Condition induite : l'autorisation des dirigeants de la verrerie qui s'y retrouvent, palliant en partie par ce biais le déficit de formation. Toute recrue n'accède pas nécessairement au grade supérieur. La place de souffleur est enviée et s'acquiert, certes par la qualité de son savoirfaire, mais aussi, soit par libération du poste, soit par extension du site verrier, soit par création d'un nouvel établissement tant proche qu'éloigné, car le verrier n'hésite pas à proposer ses services au meilleur offrant, pratiquant le nomadisme.

Toute pièce façonnée étant payée à l'ouvrier verrier, quel intérêt a ce dernier à produire des objets à titre personnel? Aucun, à en croire

18. Entretien avec Noëlle Gérôme, Mortagne-sur-Gironde (Charente-Maritime), 25 juin 2012. Nous tenons à remercier celle-ci pour les éclairages qu'elle a pu nous offrir.

19. À la fin du XIX siècle à Baccarat, la demi-heure ainsi monopolisée est autant de manque au rendement de chaque place, rappelle Elisabeth Cibot-Genin, mais, citant le directeur de l'époque, elle ajoute que «le chef de place reste libre d'arrêter cette pratique dès lors qu'elle peut être préjudiciable au bon fonctionnement de la place»; Elisabeth Cibot-Genin, "Création et innovation chez Baccarat et Daum...", op. cit., p. 274, note 27. 
Yvon Censier, ancien verrier issu d'un long lignage d'ouvriers en verre, ou encore Marcel Tajana, ancien directeur des verreries de Blérancourt (Aisne), puis de Souvigny (Allier) après un passage par Le Nouvion-en-Thiérache (Aisne $)^{20}$. Ce dernier tolérait le bousillage à condition que cela reste très raisonnable, n'hésitant pas à briser tout excès en sortie d'arche de recuit, car «l'ouvrier est là pour travailler». De son côté, le verrier bousille peu, préférant de loin augmenter la cadence de fabrication de l'équipe pour améliorer le salaire respectif de chacun puisque le mode de rétribution s'effectue "aux pièces» pour l'ensemble, puis au grade pour chaque ouvrier, le souffleur chef de place étant le mieux payé de tous. Au début du XIX e siècle, c'est ce dernier qui touche la somme globale et à qui il incombe de payer les membres de son équipe. Mais même si cela existe, le bousillage constitue très minoritairement un moyen d'améliorer son salaire, quel que soit le grade du verrier. Par ailleurs, ne s'improvise pas bousilleur qui veut. Il faut en être capable. Or, au travail, le verrier répond à des commandes de pièces. Sa créativité - quand elle est requise, ce qui est rare - comme son savoir-faire sont au service du maître de verreries.

Le XIX ${ }^{e}$ siècle avançant, mise à part l'auto-émulation, le verrier ou plutôt son équipe cherchant à battre son record de fabrication de la veille, il n'existe guère de moyen de s'exprimer, de chercher à se dépasser, d'autant que les techniques de production évoluent lentement. L'antique mode opératoire des verriers, c'est-à-dire le soufflage à la bouche au moyen d'une canne, long tube métallique, ne subira qu'une ultime phase de mutation, celle de la mécanisation totale, mécanisation en marche timide dès la fin du XIX ${ }^{\mathrm{e}}$ siècle. Seuls les souffleurs en bouteilles connaitront une phase intermédiaire, celle du semiautomatique avec la machine française Boucher de 1898. Le dernier bastion à la main, celui de la gobeleterie, "tombe» dans les années 1980. Or, le bousillage permet de démontrer son pouvoir créatif, sa capacité de «dialogue avec la matière rébarbative» en s'investissant dans une ouvre personnelle, pour reprendre les termes d'Yves Deforge ${ }^{21}$. On peut aller plus loin, avec Noëlle Gérôme, en suggérant que le bousillage représente une expression de l'hypertrophie de la liberté dans le travail ${ }^{22}$.

Véritable détournement, le bousillage peut ainsi être considéré comme un moyen de lutte personnelle contre l'appauvrissement du travail, fruit de la routine, le façonnage du verre ne subissant que peu de mutation sur la place de travail, si ce n'est celle radicale de sa mécanisation complète.

20. Entretiens avec Marcel Tajana, Souvigny (Allier), 20 mars 2012, et Yvon Censier, Aulnoye-Aymeries (Nord), 29 février et 27 mars 2012.

21. Yves Deforge, "Questions vives», postface au livre de Gilbert Simondon, Du mode d'existence des objets techniques, Aubier, 1989, p. 330; repris par Etienne de Banville, op. cit., p. 11.

22. Entretien avec Noëlle Gérôme, Mortagne-sur-Gironde (Charente-Maritime), 25 juin 2012. 


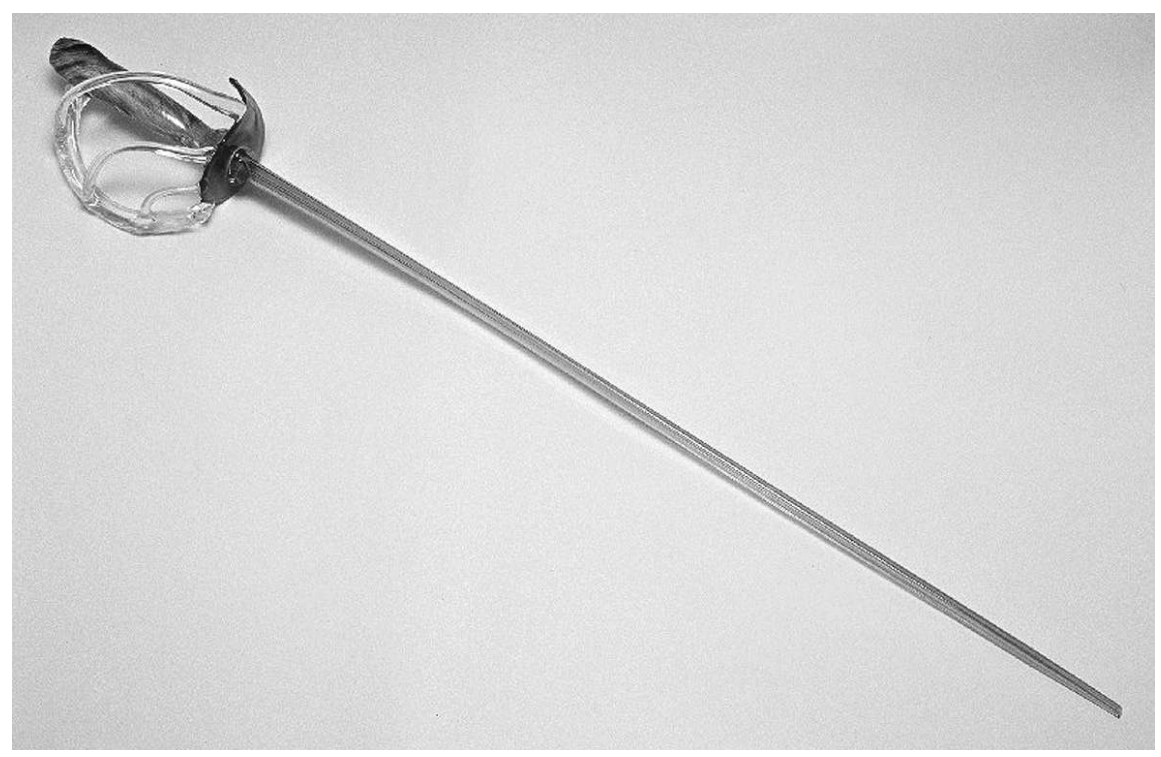

Fig. 2 : Anonyme, épée en verre, fin XIX ${ }^{\mathrm{e}}$ - début XX $\mathrm{XX}^{\mathrm{e}}$ siècle; Collection du Musée-atelier départemental du verre à Sars-Poteries - Conseil Général du Nord, inv. n 967.55.7; cliché (C) Philippe Beurtheret. Cet objet n'a d'utile que de représenter un symbole fort de l'ancienne noblesse du verre.

\section{UN REMÈDE À L'APPAUVRISSEMENT DU TRAVAIL}

De son savoir-faire, le verrier tire son prestige social. Mais que reste-t-il du maître-verrier noble de l'Ancien Régime? Ầ mesure que le XIX ${ }^{\mathrm{e}}$ siècle s'avance, il devient peu à peu une machine humaine dépossédée de son savoir-faire.

La haute technicité du travail verrier a amené l'anoblissement de son détenteur, lequel transforme du sable en produit à forte valeur ajoutée. Les princes attirent donc ces techniciens dans leurs états en leur octroyant très tôt la noblesse, monopole personnel transmis par filiation et conférant une exemption fiscale assez large. Avec l'enregistrement de l'Armorial général de France, le technicien ne peut plus être anobli, sauf exception. Mais le maîtreouvrier roturier travaille d'égal à égal avec le maître-verrier noble. Passée la Révolution, l'un comme l'autre deviennent maîtres-souffleurs, parmi les mieux payés de ce monde ouvrier qui ne cesse de grossir avec l'industrialisation de la première moitié du XIX $\mathrm{X}^{\mathrm{e}}$ siècle. Or, l'explosion des créations de verreries bouscule l'ordre des choses, les subalternes étant propulsés souffleurs à la tête de la multitude des nouvelles équipes. L'homme du verre perd peu à peu de sa superbe : «maitre-souffleur» au début de la Révolution industrielle, il est "ouvrier verrier" au milieu du XIX siècle, puis simple "verrier» dès le dernier tiers. S'il maîtrise son art, cela ne fait plus de lui un maître, mais un 


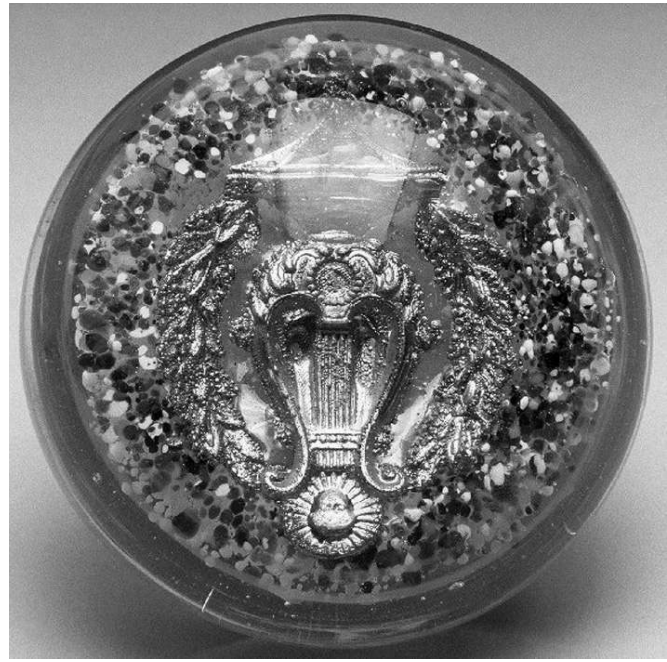

Fig. 3 : Anonyme, presse-papiers à insigne de musique, fin XIX ${ }^{\mathrm{e}}$-début $\mathrm{XX}^{\mathrm{e}}$ siècle; Collection du Musée-atelier départemental du verre à Sars-Poteries - Conseil Général du Nord, inv. $n^{\circ}$ 967.30.13; cliché (C) Philippe Beurtheret.

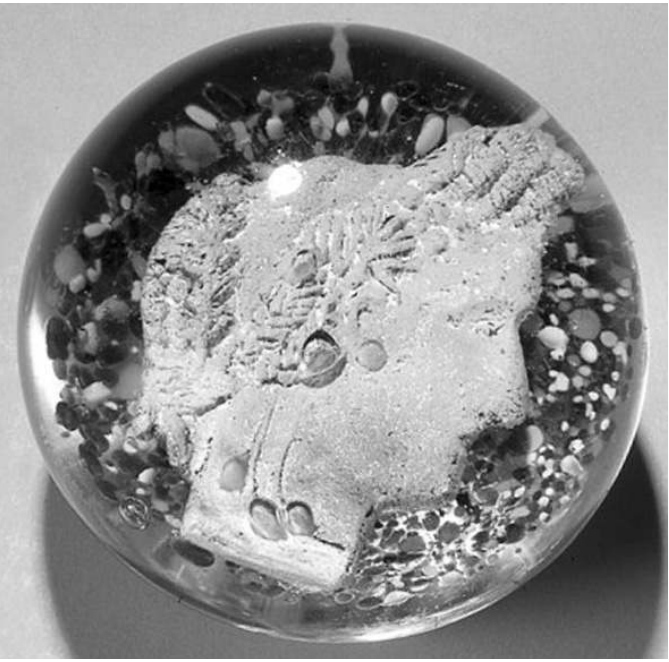

Fig. 4 : Anonyme, presse-papiers à tête de femme, fin $\mathrm{XIX}^{\mathrm{e}}$ - début $\mathrm{XX}^{\mathrm{e}}$ siècle; Collection du Musée-atelier départemental du verre à Sars-Poteries - Conseil Général du Nord, inv. $n^{\circ}$ 967.30.12; cliché (C) Paul Louis.

exécutant. La pratique du bousillage représente alors un moyen de reconnaissance envers les siens mais aussi plus largement au sein du prolétariat. Aussi c'est par l'objet que nous nous proposons ici d'appréhender les relations entre homme et technique. Au-delà de la mobilisation des compétences, il existe un véritable langage de ces objets bousillés.

Pièce prestigieuse et emblématique, le presse-papiers est l'exemple-type de la réappropriation personnelle du métier. Modèle commercial très en vogue au milieu du XIXe siècle, sa mode perdure dans une moindre mesure jusque dans les années 1880, époque où le bousillage est en pleine floraison. Réalisable seul, son façonnage est alors repris à son compte par le verrier, l'objet ne figurant plus dans les catalogues d'usine. Le plus délicat n'est pas d'inclure des grains de verre de couleurs diverses sur un fond de verre, ni de recouvrir le tout d'une couche de verre blanc transparent, mais d'y inclure par-dessus un décor complémentaire, insigne de musique (métal; fig. 3) ou tête de femme (cristallo-cérame ou composé de terres réfractaires fines; fig. 4), avant que de prélever une dernière masse de verre pour couvrir le tout. Le verrier, à l'aide d'un instrument en bois en creux préformé, «mailloche» enfin l'ensemble afin d'obtenir une forme égalisée. Toute inclusion ne "corde» pas nécessairement avec le verre support, générant des tensions internes parfois fatales à l'objet.

Moins techniques, abondent les "glettes" (fig. 5). Contraction du mot galette ou dérivé de l'allemand gleiten, glisser, ce petit palet rond sert à jouer 


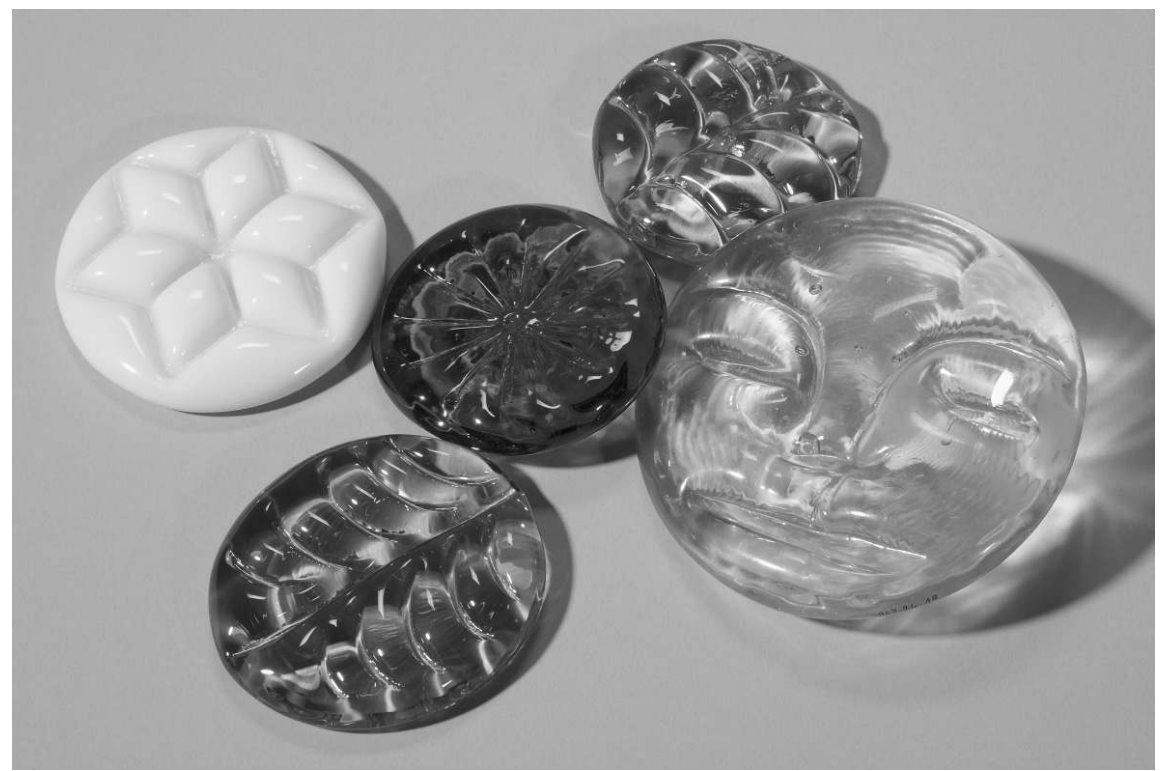

Fig. 5 : Anonymes, glettes, fin XIX $-\mathrm{XX}^{\mathrm{e}}$ siècle; Collection du Musée-atelier départemental du verre à Sars-Poteries - Conseil Général du Nord, inv. de gauche à droite ${ }^{\text {os }}$ 967.24.1, 967.24.6, 967.24.2, 967.24.18 et 967.24.4; cliché (C) Paul Louis. Reprenant une technique de formage identique, la glette à visage humain (à droite) date du dernier tiers du XXe siècle.

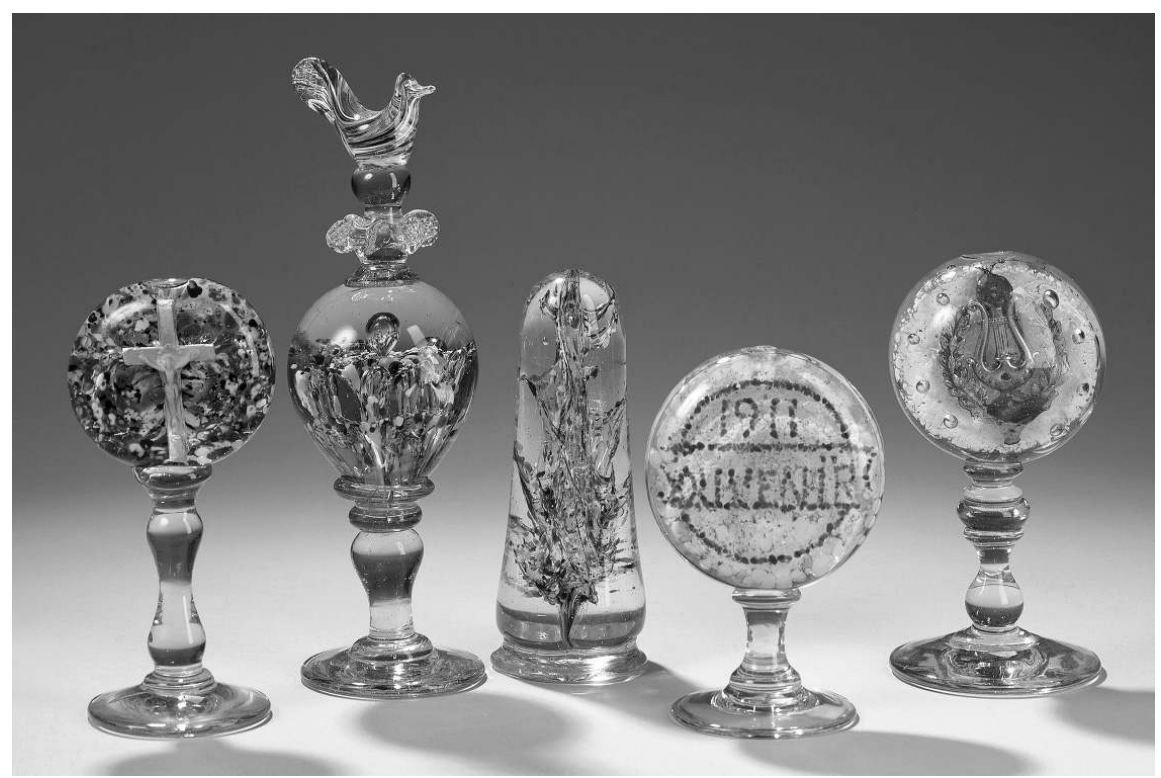

Fig. 6 : Anonymes, presse-papiers à inclusions diverses, fin $\mathrm{XIX}^{\mathrm{e}}$-début $\mathrm{XX}$ e siècle; Collection du Musée-atelier départemental du verre à Sars-Poteries - Conseil Général du Nord, inv. de gauche à droite $\mathrm{n}^{\text {os }}$ 967.32.6, 967.32.4, 967.30.14, 967.32.1 et 967.32.7; cliché (C) Paul Louis. 
à la marelle ou "paradis". Le verrier n'hésite pas à imprimer sur ce bout de verre chaud qu'il coupe et laisse s'étaler, un décor à l'aide du premier outil à portée de main, fers de tranchage, paire de pincettes, cachet comme pour le sceau de cire... Les combinaisons sont multiples et font le bonheur des enfants de verriers et au-delà. Vient ensuite la gourde, paraison de verre, bulle primaire soufflée au bout de la canne (fig. 6). Or, cette gourde en dit long sur l'évolution des habitudes de métier. À l'origine, les souffleurs étanchaient leur soif sur le compte du maitre de verreries. Puis, ils eurent à fournir en boissons leur équipe. Enfin la tradition s'est perdue, individualisant davantage le verrier. Â la fin du XIX $\mathrm{XI}^{\mathrm{e}}$ siècle, l'homme du verre est connu pour être grand consommateur de boissons - pas nécessairement d'alcool. La gourde demeure un objet démonstratif, car de petites dimensions, ce qui contraste avec les nécessités de se désaltérer compte tenu de l'ardeur du four de fusion $\left(1200^{\circ} \mathrm{C}\right)$ et de la pénibilité de l'ouvrage.

Au travers du bousillage, le rapport à l'objet est modifié. Chaque œuvre constitue une infime partie de la réappropriation consciente ou inconsciente du métier du verre, personnelle et collective.

\section{UNE RÉAPPROPRIATION COLLECTIVE DU MÉTIER}

N'importe quel verrier peut bousiller et certains types d'activités verrières semblent des champs plus favorables que d'autres à son expression. Somme toute, chacun participe d'un ensemble de réappropriation collective du métier.

Le bousillage se pratique dans tous les domaines de la verrerie, flaconnage, gobeleterie, bouteillerie, verre à vitres, toutefois seuls les deux premiers champs d'activités sont davantage propices, car le verrier y dispose de verre de couleurs variées. Celui de Sars-Poteries, dont nous présentons quelques bousillages pour accompagner notre propos, était spécialisé dans le flacon et le service de table. Pourtant, en dépit du faible nombre de couleurs et d'une moindre intensité du matériau, le verrier en bouteilles s'adonne lui aussi au bousillage. Ne s'improvise pas bousilleur qui veut. En gobeleterie, un bon "poseur de jambes» ne fait pas nécessairement un bon bousilleur. De même en bouteillerie pour un bon "embouchurier». Le verre est un liquide surfondu qui semble aisé à manipuler, mais qui refroidit vite. Tous les verriers ne bousillent donc pas et, au final, les volumes bousillés demeurent donc insignifiants en comparaison des tonnages de production industrielle. Bousiller, c'est exposer une part de soi et de sa technique, puis s'exposer à la critique des chefs et de ses camarades de travail, l'objet bousillé étant à la vue de tous à la sortie d'arche de recuit. À l'image de la perruque d'Étienne de Banville, le bousillage représente une "création sous contrainte» de temps, 


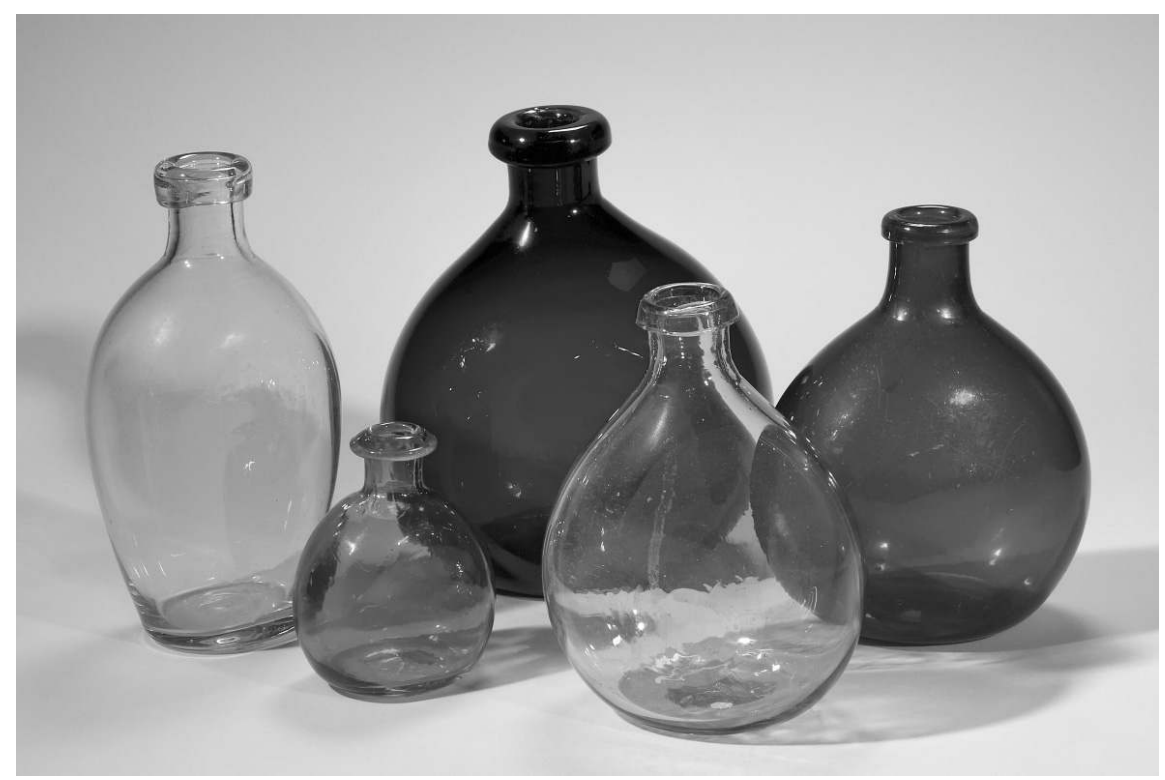

Fig. 7 : Anonymes, gourdes, fin XIX $\mathrm{X}^{\mathrm{e}}$ - début $\mathrm{XX}^{\mathrm{e}}$ siècle; Collection du Musée-atelier départemental du verre à Sars-Poteries - Conseil Général du Nord, inv. de gauche à droite $\mathrm{n}^{\text {os } 967.15 .28,967.15 .9,967.15 .6, ~ 967.15 .4 ~ e t ~ 967.15 .1 ; ~ c l i c h e ́ ~() ~ P a u l ~ L o u i s . ~}$

de matériau, du regard de l'autre, du savoir-faire acquis et du nombre de personnes disponibles.

Le verrier peut, seul, bousiller glette, gourde ou presse-papiers. Mais dès que la forme se complique, il a besoin d'aide. À chaud, il lui faut un servant pour lui apporter du verre. Ici, pour poser une cordeline qu'il modèlera en collerette; là, pour ajouter jambe et pied à un presse-papiers (fig. 7). Ce travail s'effectue avec fourniture d'une contrepartie matérielle, c'est-à-dire en échange d'une pièce, et/ou cognitive, par le partage d'un tour de main. À froid, à l'image de la lampe Imbert-Deharveng, le bousillage franchit les portes de la halle aux fours pour gagner l'atelier de taillerie où il dépend des bons soins d'un tailleur et de sa meule de pierre, avec contrepartie. Parfois, tel le verre de mariage, le bousillage reçoit des inscriptions exécutées par un graveur sur verre. Cela permet une utilisation pleine de l'usage symbolique du verre. Au-delà de l'objet dans sa simple expression physique, existe un phénomène de halo, celui décrit par Gilbert Simondon : «l'objet technique laisse rayonner autour de lui une lumière qui dépasse sa réalité propre et se répand sur l'entourage : il y a ainsi une zone de technicité plutôt qu'un objet technique; c'est la technicité de l'objet qui rayonne»²3. La virtuosité

23. Gilbert Simondon, «L'effet de halo en matière technique : vers une stratégie de la publicité», Cahiers de l'Institut de science économique appliquée, série M, $\mathrm{n}^{\circ} 7$, mars 1960; repris dans Les Cahiers philosophiques, $\mathrm{n}^{\circ}$ spécial Gilbert Simondon, CNDP, $\mathrm{n}^{\circ}$ 43, juin 1990, p. 10-11; cité par Étienne de Banville, op. cit., p. 13. 
technique devient instrument de distinction pour celui qui a réalisé un bousillage complexe. Cette complexité ne saute pas nécessairement aux yeux des non-initiés. Un simple bouton creux au milieu d'une jambe de guéridon représente parfois bien davantage que l'inclusion de grains de verre de couleur; inclusion qui est signe distinctif d'un bousillage, mais qui n'en est cependant pas l'unique moyen de reconnaissance.

Le maittre de verreries gagne au bousillage la formation de son ouvrier. Tout au plus y laisse-t-il un peu de matière, et encore, si le bousillage n'aboutit pas, le verre est mis à la récupération pour sa refonte. Bousiller se traduit d'ailleurs outre-Manche par to make rubbish, faire du déchet, et bousillage outre-Rhin par geschundenesglas, schund signifiant rebut. Cela s'explique. Lorsque le jeune ouvrier débute, il est loin d'être capable de réaliser d'emblée une belle pièce, ce qui donne immanquablement une impression de travail inachevé, lequel, compte tenu des contraintes propres à ce matériau, peut être fragile sous l'effet de la libération des tensions internes. Toutefois, le bousillage ne constitue pas qu'un "objet maladroit de l'apprenti». Même le plus expérimenté des verriers fait du rebut lorsqu'il cherche à réaliser une pièce particulière, d'autant qu'il dispose de peu de temps. Seul le temps de pause ou celui de fin de journée est disponible pour le bousillage. Or, la "braise» ou pause dure peu, l'heure accordée se répartissant en trois volumes horaire : quinze minutes, une demi-heure et quinze minutes, ou trois fois vingt minutes. Quant à la fin de journée, autour du four de fusion à pots, dans la demi-heure qui suit l'arrêt officiel du formage, le «renfourneur» emplit chaque creuset de fusion de composition vitrifiable fraîche. Et que dire d'un four à bassin à fusion continue où le verre est travaillé sans relâche jour et nuit des mois durant et où les équipes se succèdent les unes aux autres. Il faut donc se hâter en bousillant.

Or, bousiller pourrait provenir de la déformation du verbe patoisant bersiller, réfléchir. Même si le verrier maîtrise le façonnage de divers types de pièces commerciales, il est obligé de réfléchir avant que de vouloir donner forme à ce qu'il cherche à exécuter. Mais en a-t-il le temps? La pause est de courte durée et le verre refroidit très vite. Soit il multiplie les rebuts, soit il répète des formes pour la réalisation desquelles il se sent à l'aise. Jusqu’à quel point peut-il porter son bousillage d'une seule pièce, d'un seul tenant? Jusquaux limites des dimensions du four de recuit. Ainsi en est-il de l'épée (fig. 2), objet symbolique et prouesse technique, car plus l'objet est imposant, plus il est difficile d'en maintenir la forme et une température uniforme afin d'éviter qu'il ne "pète", selon le jargon du métier. Par ailleurs, lorsque que le jeune verrier débute, la crainte du chef de four est de voir celui-ci laisser tomber du verre de couleur, par exemple une couleur foncée dans un pot en contenant une autre plus claire rendrait le verre ainsi souillé impropre au façonnage pour toute une équipe. C'est là que nous rejoignons l'explication de Charles Grangagnage, lequel fait, en 1845, de bousiller un verbe issu du 


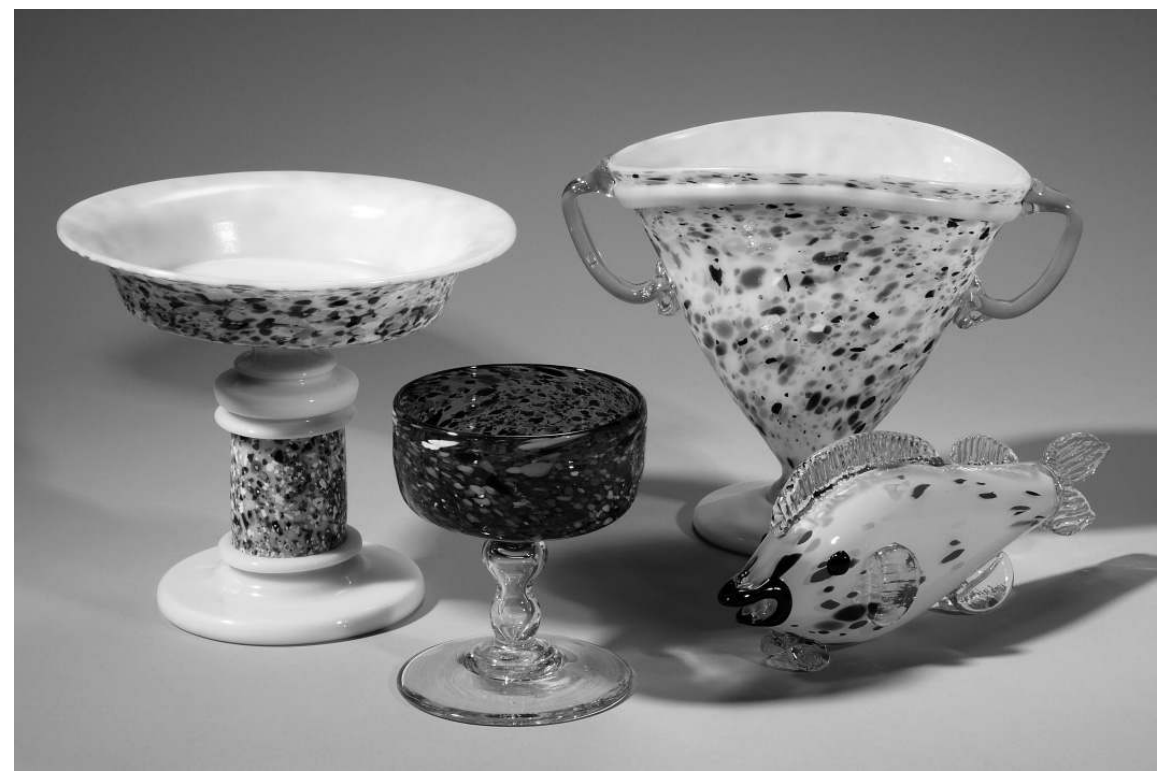

Fig. 8 : Anonymes, ensemble de bousillages, fin $\mathrm{XIX}^{\mathrm{e}}-\mathrm{XX}^{\mathrm{e}}$ siècle; Collection du Musée-atelier départemental du verre à Sars-Poteries - Conseil Général du Nord, inv. de gauche à droite $\mathrm{n}^{\text {os }}$ guéridon 967.5.26, sucrier 967.2.11, vase 967.19.47 et poisson 967.9.7; cliché (C) Paul Louis.

wallon brodi, faire quelque chose vite et mal, tiré du hollandais brodden, broddelen, bousiller, lui-même émanation de l'ancien allemand signifiant souiller ${ }^{24}$. Si l'inclusion de grains de verre de couleur dans le verre opale blanc de la coupe et de la colonne d'un guéridon (fig. 8 , à gauche) participe à la dépréciation d'un modèle commercial, cela a demandé réflexion et savoirfaire. Il en va de même pour une simple fleur. Il y a peu de place à l'improvisation, même si le spectateur néophyte en retire cette impression n'imaginant pas que soit concentré là l'aboutissement de plusieurs décennies de formation personnelle, d'échanges intergénérationnels, que de réappropriation collective d'un métier.

Véritable phénomène social propre au monde verrier, le bousillage est pourtant une activité méconnue, desservie d'une part par un manque de connaissances techniques et, d'autre part, par un terme qui masque sa réalité. Il représente toutefois à l'échelle de chaque verrier l'aboutissement d'une longue suite de tâtonnements dans la manipulation d'un matériau incandescent,

24. Charles Grangagnage, Dictionnaire étymologique de la langue wallonne, Liège, Félix Oudart, 1845, p. 79 . 
de son apprentissage. Le bousillage se propage en France dans le dernier tiers du XIX ${ }^{e}$ siècle, à l'époque même où le verrier sent qu'il devient de plus en plus une machine humaine, de plus en plus dépossédé de son savoir-faire. Véritable détournement sur "place», le bousillage, né de l'appauvrissement du travail, démontre la réappropriation collective de cet art. Pour les plus complexes d'entre les objets bousillés, cela aura nécessité l'accord du maitre de verreries, du chef de four, d'un ou plusieurs collègues de travail, du personnel de relevage d'arche de recuit, d'un tailleur voire d'un graveur sur verre. Création sous contraintes, cette somme de travail ne transforme-t-elle pas un bousillage en un chef-d'œuvre, même si en matière de bousillage comme de perruque, les protagonistes s'en défendent. Pourtant, si les compétences mises en jeu sont silencieuses, les objets conservés dans les collections, pour peu qu'on les observe avec attention, en témoignent.

Stéphane Palaude est docteur en histoire de l'Université de Lille III et président de l'Amaverre. 